\title{
Nucleic Acid Crystallization and Phase Determination Facilitated by Selenium Functionalization
}

Xinghua Chen, Cen Chen, Jianhua Gan, Wen Zhang, Oksana O. Gerlits, Jozef Salon, Julianne Caton-Williams, Sibo Jiang, Hehua Liu, and Zhen Huang*, Professor and Director of SeNA Research Laboratory, Department of Chemistry, Georgia State University, Atlanta, GA 30303, and SeNA Research Inc.; Email: Huang@gsu.edu

Selenium atom-specific functionalization can offer nucleic acids with many unique and novel properties (such as facilitated crystallization and phase determination) without significant perturbation of 3D structures of nucleic acids and their protein complexes. Nucleic acids possess not only the ability to store genetic information and participate in transcription and translation, but also the capacity to adopt well-defined 3D structures, which can be readily adjusted to meet various functional needs (such as catalysis and therapeutics). Although the importance of numerous nucleic acids in catalysis, gene expression, protein binding and therapeutics has been acknowledged by the entire scientific society, current understanding of nucleic acid-protein functions and structures is still limited, especially highresolution structures. This novel Se-atom-specific functionalization will provide important tools to investigate nucleic acid structure/folding, recognition and catalysis, to study nucleic acids and their protein interactions, to improve biochemical and biophysical properties of nucleic acids, and to explore potential nucleic acid therapeutics and diagnostics. Our presentation will focus on the most recent selenium-atom functionalization of nucleic acids and their potential applications in 3D structure-and-function studies and anticancer therapeutics in molecular medicine.

Work is supported by NIH (R01GM095881, GM095086, ES026935) and NSF (MCB-0824837 \& CHE-0750235).

\section{Selected Publications:}

1. Jing Zhang, Hehua Liu, Qingqing Yao, Xiang Yu, Yiqing Chen, R. Cui, B. Wu, L. Zheng, J. Zuo, Zhen Huang*, Jinbiao Ma* and Jianhua Gan*,"Structural basis for single-stranded RNA recognition and cleavage by C3PO”, Nucleic Acids Research, 2016, 44, 94949504.

2. Liqin Zhang, Zunyi Yang, Sefah, Bradley, Hoshika, M.-J. Kim, H.-J. Kim, Zhu, Sena Cansiz, I-Ting Teng, Carole Champanhac, Christopher McLendon, Chen Liu, Wen Zhang, Dietlind L. Gerloff ${ }^{3}$, Zhen Huang", Weihong Tan" and Steven A. Benner", "Crystal Structure, Evolution, and Function of Six-Nucleotide DNA. Exploring its Large Sequence Space”, Journal of American Chemical Society, 2015, 137, 6734-6737.

3. Rob Abdur, O. Gerlits, Jianhua Gan, J. Jiang, J. Salon, A. Kovalevsky, A. Chumanevich, I. Weber, Zhen Huang*, "Novel Complex MAD Phasing and RNase H Structural Insights by Selenium Oligonucleotides", 2014, Acta Crystallographica Section D, 2014, D70, 354-361.

4. Jia Sheng, Jianhua Gan, Alexie Soars, Jozef Salon and Zhen Huang*, "Structural Insights of Non-canonical U•U Pair and Hoogsteen Interaction Probed with Se Atom", Nucleic Acids Research, 2013, 41, 10476-10487.

5. Huiyan Sun, et. al., Liu, Zhen Huang*, "2-Selenouridine Triphosphate Synthesis and Se-RNA Transcription”, RNA, 2013, 19, 13091314.

6. Jozef Salon, Jianhua Gan, Rob Abdur, Hehua Liu and Zhen Huang*, "Synthesis of 6-Se-Guanosine RNAs for Structural Study", Organic Letter, 2013, 15, 3934-3937.

7. Wen Zhang, Abdalla E. Hassan, and Zhen Huang*, "Synthesis of Novel Di-Se-containing Thymidine and Se-DNAs for Structure and Function Studies", Science China: Chemistry, 2013, 56, 273-278.

8. 2. Huiyan Sun, Jia Sheng, Abdalla E. A. Hassan, Sibo Jiang, Jianhua Gan and Zhen Huang*, "Novel RNA Base Pair with Higher Specificity using Single Selenium Atom”, Nucleic Acids Res., 2012, 40, 5171-5179.

9. Jia Sheng, Wen Zhang, Abdalla E. A. Hassan, Jianhua Gan, Alexei Soares, Song Geng, Yi Ren, Zhen Huang*, "Hydrogen Bond Formation between the Naturally Modified Nucleobase and Phosphate Backbone", Nucleic Acids Research, 2012, 40, 8111-8118.

10. Wen Zhang, Jia Sheng, Abdalla E. Hassan, and Zhen Huang*, "Synthesis of Novel 2'-Deoxy-5-(Methylselenyl)Cytidine and SeDNAs for Structure and Function Studies", Chemistry-An Asian Journal, 2012, 7, 476-479.

11. Lin, L.; Sheng, J.; Huang, Z. Chemical Society Reviews, 2011, 40, 4591.

12. Sheng, J.; Hassan, A. ; Zhang, W.; Zhou, J.; Xu, B.; Soares, A. S.; Huang, Z. Nucleic Acids Res., 2011, 39, 3962. 